\title{
Digital Integrator for Fast Accurate Measurement of Magnetic Flux by Rotating Coils
}

\author{
Pasquale Arpaia, Alessandro Masi, and Giovanni Spiezia
}

\begin{abstract}
A fast digital integrator (FDI) with dynamic accuracy and a trigger frequency higher than those of a portable digital integrator (PDI), which is a state-of-the-art instrument for magnetic measurements based on rotating coils, was developed for analyzing superconducting magnets in particle accelerators. Results of static and dynamic metrological characterization show how the FDI prototype is already capable of overcoming the dynamic performance of PDI as well as covering operating regions that used to be inaccessible.
\end{abstract}

Index Terms-Converters, magnetics, particle accelerator science, signal analysis, signal processing.

\section{INTRODUCTION}

$\mathbf{F}$ OR THE Large Hadron Collider (LHC), the particle accelerator under construction at the European Organization for Nuclear Research (CERN), Genève, Switzerland, accurate dynamic measurements of a magnetic field are necessary to implement a suitable control of trajectories as well as to focus the beam correctly. The most accurate technique for measuring magnetic flux is based on a calibrated coil, rotated at a typical speed of $1 \mathrm{r} / \mathrm{s}$ inside the magnet core, whose output voltage signal is integrated digitally [1]. The flux increment is measured between two angular positions, provided by an encoder mounted on the coil shaft triggering a digital integrator. Until now, the portable digital integrator (PDI) [2] has been used as the de facto standard at CERN and in other nuclear research laboratories. However, for the LHC and other more demanding applications, a new generation of rotating coils with higher rotation speeds up to $10 \mathrm{r} / \mathrm{s}$ is under development at CERN [3], [4]. It will allow a larger bandwidth up to $50-100 \mathrm{kHz}$ and a higher target dynamic accuracy up to $10 \times 10^{6}$ on the flux. The PDI is based on a first-order delta-sigma $\Delta-\Sigma$ modulator, and its performance gets worse when the oversampling ratio (OSR)

Manuscript received July 11, 2006; revised November 10, 2006. This work was supported by the European Organization for Nuclear Research (CERN) through Agreement K1201/AT/LHC with the Department of Engineering, University of Sannio.

P. Arpaia is with the Department of Engineering, University of Sannio, 82100 Benevento, Italy, and also with the Magnet Measurement and Tests (AT-MTN) Group, Department of Accelerator Technologies, European Laboratory for Nuclear Research (CERN), 1211 Genève, Switzerland (e-mail: arpaia@unisannio.it).

A. Masi is with the Areas, Targets and Beams Group, Department of Accelerators and Beams, European Organization for Nuclear Research (CERN), 1211 Genève, Switzerland.

G. Spiezia is with the Department of Engineering, University of Naples Federico II, 80138 Napoli, Italy, and also with the Magnets Measurements and Tests (MTM) Group, Department of Accelerator Technology, European Organization for Nuclear Research (CERN), 1211 Genève, Switzerland.

Digital Object Identifier 10.1109/TIM.2007.890787

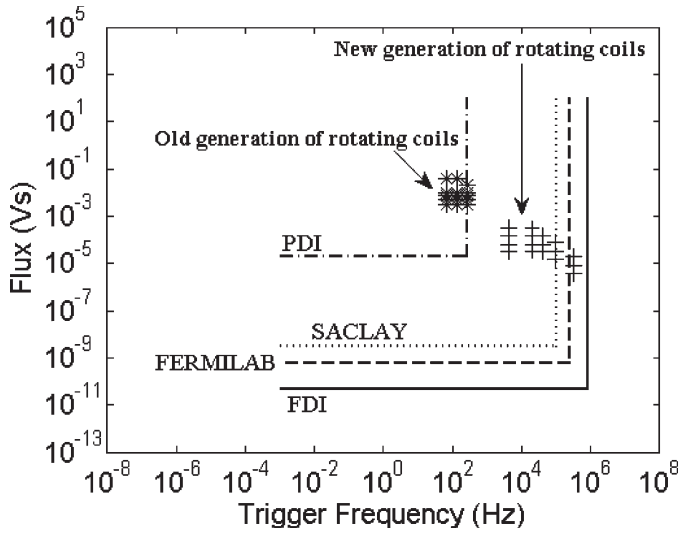

Fig. 1. Theoretical performance limits of state-of-the-art solutions and FDI, compared to working areas of old and new generations of rotating coils.

decreases, i.e., the coil trigger frequency increases. In Fig. 1, limits of ideal working areas of the PDI and other state-of-theart solutions [5], [6] related to resolution and trigger frequency are compared. The setup proposed at SACLAY acquires voltage samples with a time-stamp resolution within $5 \mathrm{~ns}$ for a final integration on a personal computer (PC) [5]. This PC-based offline integration limits the bandwidth intrinsically. At the Fermi National Accelerator Laboratory (FERMILAB), Batavia, IL, though an acquisition board with a digital signal processor (DSP) is exploited [6], the developed measuring setup was only five times faster than the PDI. Furthermore, such solutions are validated only at the conceptual level on the measuring setup, which is based on stand-alone instruments, without a standard metrological calibration, and the most critical impact of noise is not actually assessed at the board level. Moreover, offset and gain errors, predominating on the measuring chain and also requiring cyclic corrections and adjustments to a skilled operator, are not considered specifically.

In this paper, a self-calibrating self-correcting instrument, i.e., the fast digital integrator (FDI), which was conceived at CERN in cooperation with the Department of Engineering, University of Sannio, Benevento, Italy, is proposed. FDI exploits a high-rate high-resolution digital conversion, whose accuracy is not related to trigger frequency directly. In addition, it keeps the actual benefit of a larger OSR, owing to a maximum sampling rate of $800 \mathrm{kS} / \mathrm{s}$ and an online DSP-based integration. In Section II, the design proposal of the FDI measurement chain is described. In Section III, experimental results of metrological characterization of the FDI prototype are presented by comparing PDI and FDI performance. 


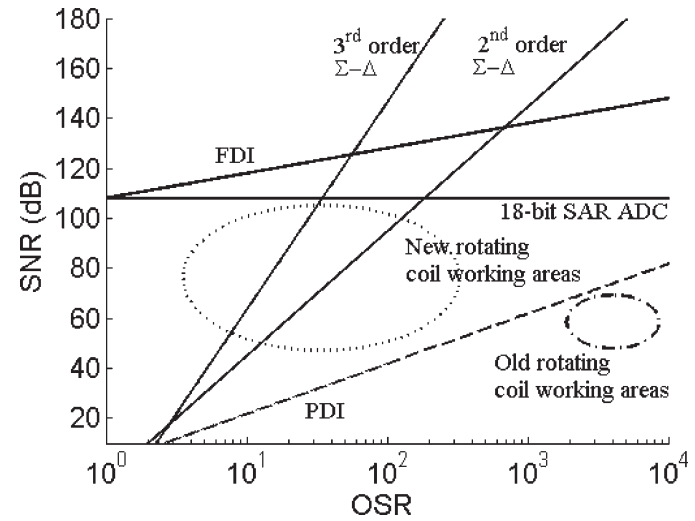

Fig. 2. Performance of an ideal PDI, typical second-order $\Delta-\Sigma$ modulator, ideal third-order $\Delta-\Sigma$ modulator, ideal 18-bit $800-\mathrm{kS} / \mathrm{s}$ SAR ADC, and ideal FDI.

\section{FDI PROPOSAL}

In this section, 1) the design strategy and 2) the architecture of the FDI are detailed.

In the design strategy of a fast accurate dynamic flux analyzer, the new generation of high-performance $\Delta-\Sigma$ modulators [7], developed for metrological applications, was considered initially. In Fig. 2, their theoretical dynamic performance is expressed in terms of signal-to-noise ratio (SNR) as a function of OSR for the sake of comparison with the PDI. As well known, the higher the modulation order is, the higher the SNR rate with the OSR becomes. However, in the current application, problems of modulator bandwidth arise (e.g., third order in Fig. 2). Although the typical performance of state-ofthe-art $\Delta-\Sigma$ in Fig. 2 performs better than the ideal PDI, at the conceptual level, it leaves the most promising application area uncovered, i.e., SNR ranging from 80 to $120 \mathrm{~dB}$ and OSR ranging from 1 to 12 . These considerations suggested to leave the advantages of delta modulation and to move toward a new generation of high-sampling-rate high-resolution analogto-digital converters (ADCs): the 18-bit 800-kS/s successive approximation register (SAR) (Fig. 2). In the FDI concept, the ADC is joined to a DSP for online computation of the flux increment and, thus, for decimating the input voltage samples so that the resulting SNR trend turns out to be more attractive. Fig. 2 shows how the high resolution supports the ideal performance, just when the OSR approaches more critical values under 12. As a matter of fact, an increase in trigger frequency in order to enlarge the flux bandwidth does not affect the FDI performance in a critical way. Theoretically, the flux sampling rate can increase up to the maximum real-time ADC sampling rate, without losing the 18-bit resolution on the voltage signal, guaranteed by the ideal SAR. On the other hand, the greater the OSR is, the better the FDI performance becomes.

In the FDI architecture, the input signal arising from the coil is conditioned by a low-noise custom differential programmable gain amplifier (PGA) (Fig. 3). It was provided by a dichotomic automatic procedure for calibration and correction of gain and offset errors. Details of such a procedure are reported in [8]. A field-programmable gate array acts as an input-output processor: it supervises the PGA operations at low level and provides the interface for the board bus peripheral component interface extensions for instrumentation. A DSP supervises the board as a whole and integrates online the data digitized by the 18-bit $800-\mathrm{kS} / \mathrm{s}$ differential SAR ADC by exploiting the trigger pulses of an encoder mounted on the shaft of the rotating coil. These pulses, coming out from the flux sampler, are measured by a time base with a resolution of $50 \mathrm{~ns}$ (Fig. 3).

\section{EXPERIMENTAL RESULTS}

The performance of the FDI prototype was compared to the PDI one by dynamic and static tests carried out according to the standard IEEE 1057-1994 on digitizing waveform recorders [9]. Both of the instruments are digital integrators; nonetheless, they can work as digitizers. In fact, the PDI is based on a firstorder $\Delta-\Sigma$ modulator, i.e., a voltage-to-frequency converter, for which the trigger frequency is inversely proportional to the PDI resolution.

In this section, 1) the measurement station, 2) the static tests, and 3) the dynamic tests are described.

A measurement station, which is based on a dc calibrator and on an ac calibrator, remotely controlled via a general purpose interface bus, was set up. A function generator was used to generate the trigger.

The static tests determine the differential nonlinearity (DNL) by measuring the actual transition levels [9]. The PDI number of bits and the least significant bit (LSB) are functions of the trigger frequency. As an example, the test result in a typical working condition of a trigger frequency of $512 \mathrm{~Hz}$ is reported. In this case, the PDI LSB is $10.24 \mathrm{mV}$, and the theoretical number of bits is equal to 9.93 , for a total number of transition levels of 975 . With a theoretical number of 18 bits, the FDI has an LSB of $38 \mu \mathrm{V}$ for a total number of transition levels of 262143 . This number is very high, and the complete test would take a very long time; thus, the DNL was computed on several groups of transition levels for the PDI and FDI. In this way, it was possible to assess the DNL in local parts of the input range. By referring to the above example, in Fig. 4(a) and (b), for the PDI and FDI, respectively, test results show a very good linearity, with a static DNL within \pm 1.5 LSB. Nonetheless, at this typical PDI trigger frequency of $512 \mathrm{~Hz}$, the FDI resolution is much higher than that of the PDI.

Dynamic tests aim to determine the signal-to-noise and distortion ratio (SINAD) and the effective number of bits (ENOB) of the instrument by taking into account the noise and the distortion of all the measurement chain. They were evaluated by the standard fast Fourier transformer (FFT) test [9]. For the PDI, the SINAD was evaluated at different trigger frequency values with a sine wave input of $V_{\mathrm{rms}}=3 \mathrm{~V}$ at a frequency ranging from 10 to $120 \mathrm{~Hz}$. A typical amplitude spectrum is shown in Fig. 5(a). The values of signal and trigger frequency were adjusted in order to achieve coherent sampling conditions.

For the FDI, tests were carried out with a sine wave input of $V_{\mathrm{rms}}=3 \mathrm{~V}$ at a frequency ranging from 10 to $250 \mathrm{~Hz}$. The typical amplitude spectrum of a digitized sine wave at the same frequency of $120 \mathrm{~Hz}$ is depicted in Fig. 5(b). For the sake of sampling coherence, the trigger frequency selected was equal to $25 \mathrm{kHz}$, which was higher than the one considered for 


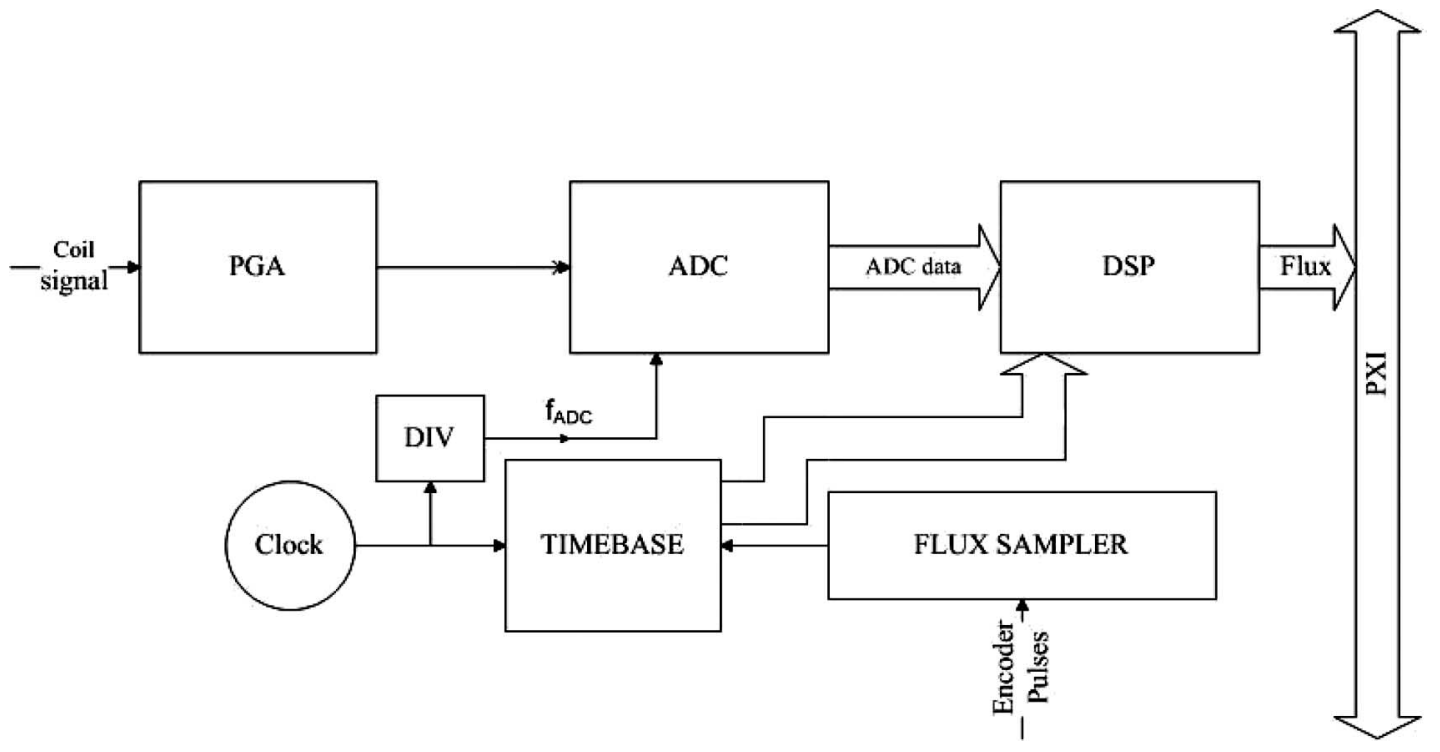

Fig. 3. FDI functional block diagram. PGA is the programmable gain amplifier.

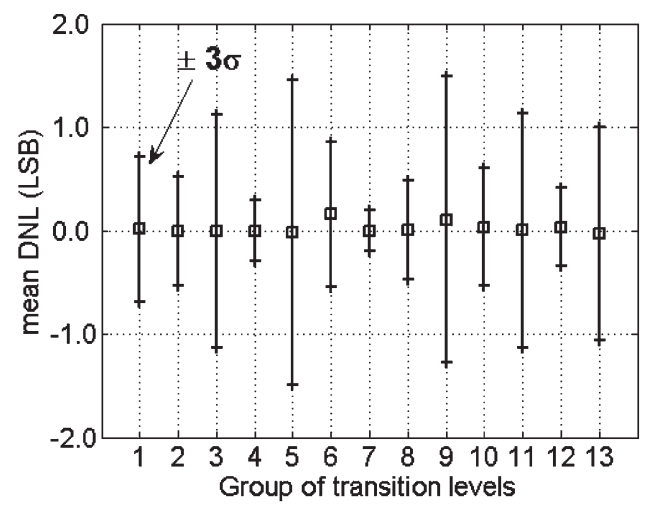

(a)

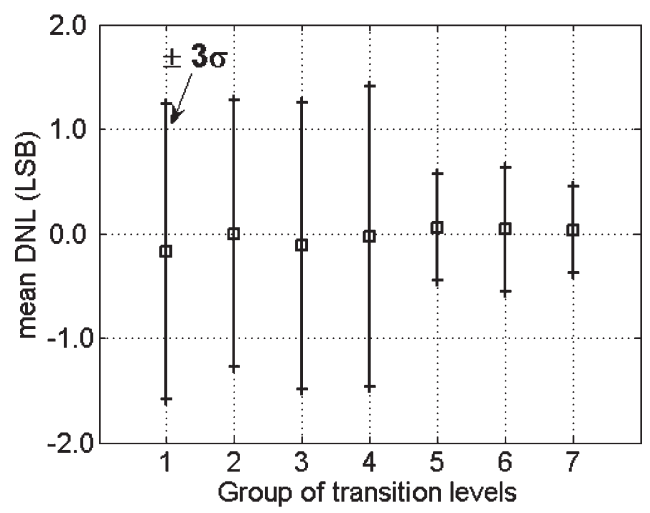

(b)

Fig. 4. (a) PDI and (b) FDI differential nonlinearity in different regions of the input range: Mean and uncertainty for each transition level group.

the PDI test, giving rise to heavier working conditions for the FDI in any case.

The SINAD, the signal nonharmonic ratio (SNHR), the total harmonic distortion (THD), the spurious free dynamic range (SFDR) [10], and the ENOB for the PDI and FDI are summarized in Table I as follows: 1) PDI ENOB turns out to be around

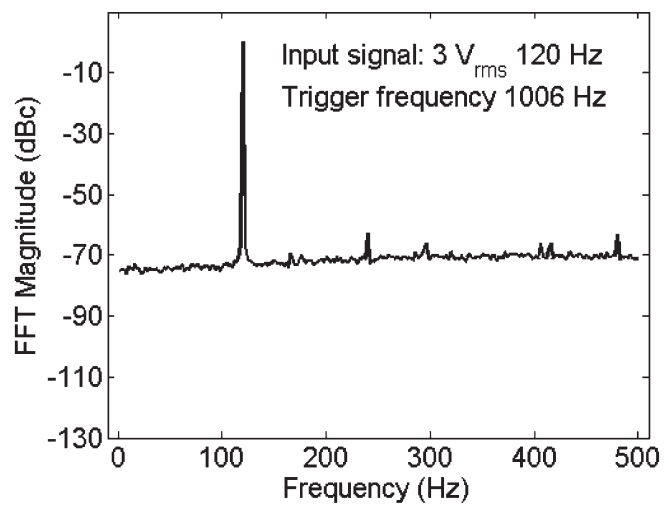

(a)

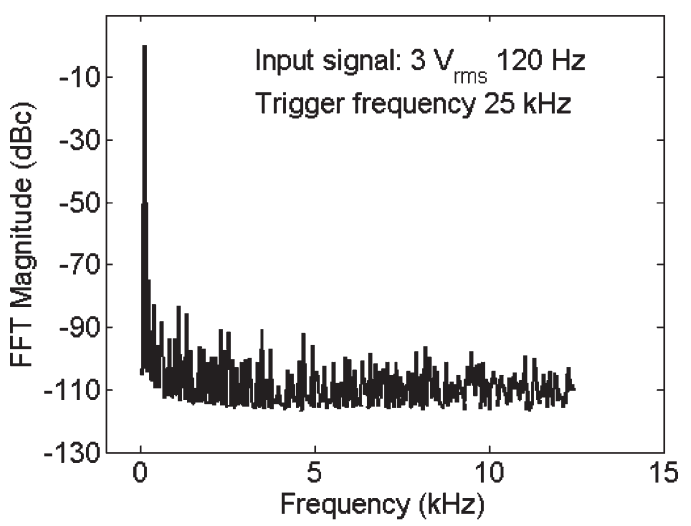

(b)

Fig. 5. Amplitude spectrum of (a) PDI and (b) FDI for an input frequency of $120 \mathrm{~Hz}$.

9 bits at a trigger frequency of $1024 \mathrm{~Hz}$, whereas 2) FDI ENOB is about 12 bits on a bandwidth of $25 \mathrm{kHz}$.

In Fig. 6, a more comprehensive comparison between the FDI and PDI performance is shown: The SINAD is reported as a function of the OSR [Fig. 6(a)] and of the trigger frequency [Fig. 6(b)]. The FDI allows a higher accuracy to be 
TABLE I

DyNAMic PERFormance of PDI AND FDI, FOR A Sine WAVE INPUT $V_{\mathrm{rms}}=3 \mathrm{~V}$ AT $120 \mathrm{~Hz}$

\begin{tabular}{|c|c|c|c|c|c|}
\hline & SINAD (dB) & SNHR (dB) & SFDR (dB) & THD (dB) & ENOB \\
\hline PDI & 47.1 & 47.4 & 62.8 & -59.5 & 7.8 \\
\hline FDI & 72.4 & 79.1 & 74.8 & -74.2 & 12.0 \\
\hline
\end{tabular}

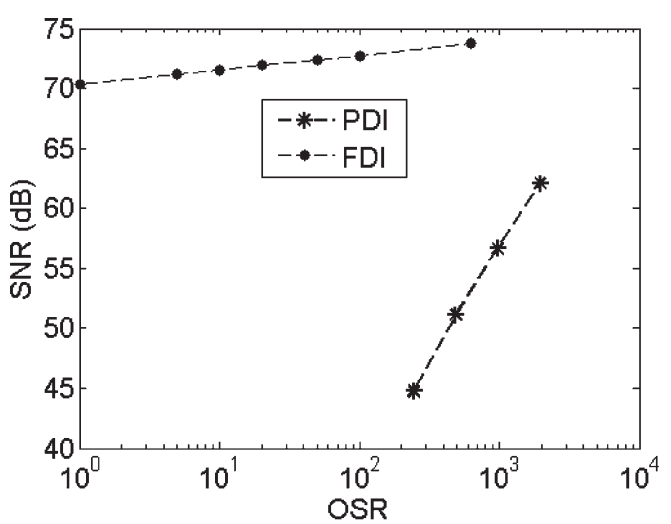

(a)

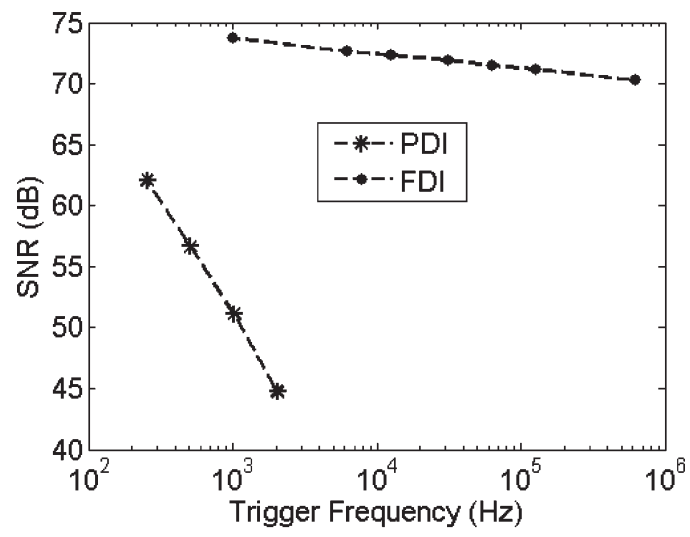

(b)

Fig. 6. FDI and PDI performance: SNR as a function of (a) OSR and (b) trigger frequency.

reached, even at a high trigger frequency where the PDI cannot operate. However, the benefits of the oversampling for the FDI prototype do not correspond to the expected ones, because the in-bandwidth nonlinearity decreases the SINAD. Since the static test showed a very good linearity, the sources of this dynamic nonlinearity must be investigated in order to improve performance further.

\section{CONCLUSION}

An FDI was conceived and prototyped at CERN to satisfy the more demanding requirements of magnetic flux measurements arising from advanced LHC applications. A metrological characterization of both a PDI and a state-of-the-art FDI was carried out. The static test showed that both PDI and FDI present a satisfying linearity, although the FDI resolution is much higher than that of the PDI. The digital conversion of the coil signal at a high sampling rate allows the FDI to achieve better dynamic results than the PDI. The FDI outperforms the PDI in all common working conditions and allows new operating ranges to be covered.
Further work will be devoted to increase dynamic performance by exploiting the DSP for online correction of nonlinearity as well as noise suppression.

\section{ACKNOWLEDGMENT}

The authors would like to thank F. Cennamo, L. Bottura, and L. Walckiers for their useful suggestions, D. Giloteaux for his constant technical support, as well as P. Cimmino, D. Della Ratta, V. Inglese, and S. Tiso for their precious collaboration.

\section{REFERENCES}

[1] L. Bottura and K. N. Henrichsen, "Field measurements," in Proc. CAS, Sep. 2004, pp. 118-151.

[2] P. Galbraith, Portable Digital Integrator, 1993. Int. Tech. Note 93-50 AT$\mathrm{MA} / \mathrm{PF} / \mathrm{fm}$

[3] S. Amet, L. Bottura, L. Deniau, and L. Walckiers, "The multipoles factory: An element of the LHC control," in Proc. 17th Int. Conf. MT, Genèva, Switzerland, Sep. 24-28, 2001, pp. 1417-1421.

[4] - "The multipoles factory: An element of the LHC control," IEEE Trans. Appl. Supercond., vol. 12, no. 1, pp. 1417-1421, Mar. 2002.

[5] C. Evesque, "A new challenge in magnet axis transfer," in Proc. 11th IMMW, Sep. 1999, pp. 1234-1237.

[6] G. V. Velev, R. Carcagno, J. DiMarco, S. Kotelnikov, M. Lamm, A. Makulski, V. Maroussov, R. Nehring, J. Nogiec, D. Orris, O. Poukhov, F. Prakoshyn, P. Schlabach, and J. C. Tompkins, "A fast continuous magnetic field measurement system based on digital signal processors," IEEE Trans. Applied Supercond., vol. 16, no. 2, pp. 1374-1377, Jun. 2006.

[7] P. Arpaia, F. Cennamo, P. Daponte, and H. Schumny, "Modeling and characterization of sigma-delta analog-to-digital converters," IEEE Trans. Instrum. Meas., vol. 52, no. 3, pp. 978-983, Jun. 2003.

[8] P. Arpaia, L. Bottura, P. Cimmino, D. Giloteaux, A. Masi, J. G. Perez, G. Spiezia, and L. Walckiers, "A fast digital integrator for magnetic field measurements at CERN," in Proc. IMTC, Apr. 2006, pp. 67-71.

[9] IEEE Standard for Digitizing Waveform Recorders, IEEE Standard 1057, 1994.

[10] IEEE Standard for Terminology and Test Methods for Analog-to-Digital Converters, IEEE Standards 1241, 2001.

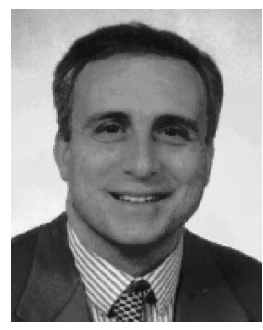

Pasquale Arpaia was born in Napoli, Italy, on February 2, 1961. He received the M.D. and Ph.D. degrees in electrical engineering from the University of Naples Federico II, Napoli, Italy.

He taught electrical and electronic measurements at the University of Naples Federico II until 2001. Then, he became an Associate Professor at the Department of Engineering, University of Sannio, Benevento, Italy. Since August 2005, he has been a Project Associate for the Large Hadron Collider at the Magnet Tests and Measurement Group, Accelerator Technology Department, European Laboratory for Nuclear Research (CERN), Genève, Switzerland. He has been a consultant on EU IV Framework Program "Standard Measurement and Testing" and evaluator for EU INTAS projects. His main research interests include ADC modeling, testing, and standardization, measurement systems on geographic networks, statistical-based characterization of measurement systems, and digital instruments for magnetic measurements in particle accelerators. In this field, he has published more than 130 scientific papers in journals as well as national and international conference proceedings.

Dr. Arpaia was a member of the Scientific and Administration Council of the new thematic University of Science and Technology. He is responsible, with Harald Schumny, for the Promoting Committee of the EUPAS Project of the IMEKO TC-4 "A/D and D/A Metrology" WG and is a Voting Member of the IEEE IM TC-10 "Waveform Measurement and Analysis" and of the IEC TC-47. He organized several international meetings in the field of Electronic Measurements and European Cooperation. He is the Editor of the subject area "Digital Instruments Standardization" for the Elsevier journal Computer Standards \& Interfaces and is an Associate Editor for the subject areas "Quality and Statistical Methods" and "Test" of the IEEE TRANSACTIONS ON EleCtronics PACKaging and ManUfacturing. 


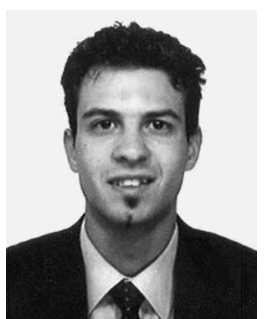

Alessandro Masi was born in Napoli, Italy, on July 19,1976 . He received the M.D. degree in electronic engineering and the $\mathrm{Ph} . \mathrm{D}$. degree in computer science from the University of Napoli Federico II, Napoli. His Ph.D. research was carried out mainly at the Magnet Tests and Measurement Group, Accelerator Technology Department, European Center for Nuclear Research (CERN), Genève, Switzerland, on high-accuracy measurement systems for superconducting magnets of the new particle accelerator, namely the Large Hadron Collider.

He has been a Researcher at the Inter-Universities Consortium of Computer Science (CINI) on the topic of network architectures for remote measurements and control systems. Since April 2005, he has been with the Lasers Photocathodes and Equipment Controls Section, Areas, Targets, and Beams Group, Accelerators and Beams Department, CERN, as a Staff Member. His main research interests include high-accuracy measurement systems for magnetic field measurement, positioning sensors, and remote control architectures.

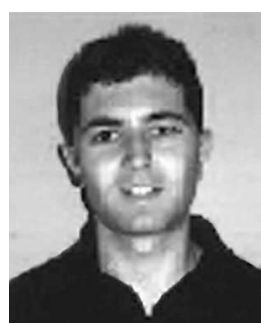

Giovanni Spiezia was born in Napoli, Italy, in January 1981 . He received the M.D. degree in electronic engineering from the University of Naples Federico II, Napoli, in March 2005. He carried out his final dissertation work with the European Organization for Nuclear Research (CERN), Genève, Switzerland, from August 2005 to December 2005 by developing a remote acquisition system for detecting high-energy proton beam impacts on the LHC collimator.

He was with the ATB Group, Department of Accelerators and Beams (AB/ATB), CERN, from April 2005 to October 2005, working on the development of an automatic test bench for step motor. He is developing his research activities on magnetic measurements with the Magnets Measurements and Tests Group, Department of Accelerator Technology (AT/ATM), CERN. He is also with the Department of Engineering, University of Naples Federico II. His main research topics are digital signal processing and magnetic measurements on particle accelerators. He has published seven papers in journals as well as in national and international conference proceedings.

Dr. Spieza won the Ph.D. competition at the Department of Electrical Engineering, University of Naples Federico II, in November 2005. 